\title{
Helical computed tomography has a role in the screening of lung cancer - The pro argument
}

\author{
Milan Patel MD FRCPC
}

$I_{d}^{n}$ n 2003, approximately 21,000 new cases of lung cancer were diagnosed across Canada. Nearly 19,000 Canadians died of this devastating disease that year (1). Worldwide, there are over one million cases identified annually (2). In the Western world, more patients die of lung cancer than from breast, prostate and colon cancer combined. Despite these figures, lung cancer has remained low on the health care agenda. In the past three decades, there has been little improvement in observed lung cancer survival rates, with overall five-year survival rates approaching $15 \%$ (3).

Since lung cancer progresses insidiously with almost no symptoms at early stages, most patients are not diagnosed until they have advanced disease with regional or distant spread. Multimodality treatment may be considered for a select group of stage 3A patients with good performance status. However, long term survival is observed in only one-quarter of patients treated in this manner (4). Treatment-related morbidity and cost are high. Treatment for patients with stages $3 \mathrm{~B}$ and IV lung cancer is palliative in the majority of cases. On the other hand, it is clear that patients with early stage, 'resectable' disease have much better outcomes. Therein lies the hope for a screening strategy.

In general, a mass screening program should be reserved for a disease that carries high morbidity or mortality, for which an effective intervention exists in the preclinical (asymptomatic) stage. Treatment in this preclinical period should yield better patient outcomes than if treatment is delayed to a symptomatic stage. The screening test itself should be accurate, safe and widely available, and targeted to an identifiable, at-risk population. Finally, the cost of the program relative to its benefit should be acceptable from a societal perspective (5). Low dose computed tomography (LDCT) scanning for lung cancer conforms to these principles.

Why is there then such trepidation in accepting LDCT as a screening tool? The failure of chest $\mathrm{x}$-ray (CXR) screening trials to show a reduction in cancer-specific mortality is undoubtedly a major reason. For example, the Mayo Lung Project randomly assigned approximately 10,000 male smokers either to intensive quarterly screening with CXR plus sputum cytology or to routine care. The experiment spanned almost one decade. At its conclusion, an interesting observation was made: the screened population had a higher incidence of lung cancer and improved survival compared with the control population, yet there was no significant difference in cancerspecific mortality (6). A detailed explanation of this paradox of improved survival with higher mortality is beyond the scope of this discussion. Critics of radiographic screening have point- ed out that the excess number of lung cancer cases seen in the screened arm was the result of diagnosing 'pseudo-disease'. That is, had these patients not been subject to a screening test, they may never have presented with symptomatic lung cancer, because some lung cancers may have a relatively benign course with very slow progression. Alternatively, this is a group of patients at increased risk for premature death due to other smoking-related illnesses, and therefore, early diagnosis of lung cancer may not have averted death from other causes. These limitations are referred to as overdiagnosis bias and lengthtime bias (5-7).

Re-examination of the CXR trials reveals major methodological flaws (7-9). For example, in the Mayo Lung Project, the 'control' arm was contaminated, with nearly $50 \%$ of patients undergoing interval CXRs. Approximately 25\% of the screened arm did not follow study protocol. The study was underpowered, with only a $20 \%$ chance of detecting a small but clinically important $10 \%$ reduction in mortality. Finally, the patients in that study were mostly actively smoking men, and the majority of cancers were squamous cell. In contrast, today, nearly one-half of patients are former smokers, often women, and adenocarcinoma is the most common type of cancer. However, the most apparent criticism is that CXR was simply the wrong screening test! CXR is quite insensitive. In the Mayo Lung Project, less than one-half of the detected lung cancers were resectable, and 30\% were pathological stage 1 . Refuting LDCT as a screening tool on the basis of historical data is not justified.

LDCT provides a low resolution image of the entire thorax in a single breath hold. Despite the 'low' resolution, nodules are detectable because of the inherent high contrast between air and tissue. Advanced image processing makes it possible to accurately determine the shape and growth of a nodule. The technology is rapidly evolving. Higher resolution images can be obtained even more quickly and with lower doses of radiation exposure. Computer-aided diagnostic technology will make automated nodule detection possible (10). An early landmark study demonstrating the promise of LDCT as a screening tool was published by the New York Early Lung Cancer Action Project (ELCAP) group in 1999 (11). This prospective, observational study enrolled 1000 asymptomatic patients over the age of 60 years into an annual LDCT screening program. Patients were current or former smokers with at least a 10 pack-year history of smoking. Nearly one-half of the patients were women. All patients underwent CXR and LDCT during the baseline (prevalence) study. LDCT detected

Divisions of Respirology and Occupational Medicine, University of Toronto, Toronto, Ontario

Correspondence: Dr Milan Patel, 2005 - 801 Bay Street, Toronto, Ontario M5S 1Y9. E-mail milan.patel@utoronto.ca 
noncalcified nodules (NCNs) in 233 patients (23\%). Only 28 patients underwent biopsy; the remainder were followed by serial CT. Of these, 27 were confirmed to be malignant and $85 \%$ (23 of 27) were pathological stage 1A. Overall, the prevalence of lung cancer was $2.7 \%$. Notably, no thoracotomy was performed for a benign nodule. Moreover, 20 of 27 cancers were not detectable by CXR. LDCT was able to detect NCNs in three times more patients than CXR, as well as four times as many lung cancers and six times as many stage $1 \mathrm{~A}$ cancers. When this cohort underwent subsequent annual screening scans, nodules were detected in only $2.5 \%$ of patients (12). Of these scan-positive patients, one-quarter (seven patients) were found to have malignancy, compared with one of 10 scanpositive cases in the initial prevalence study. With respect to costs, economic analyses using ELCAP results suggest that mass screening with LDCT may be societally acceptable, with an estimated incremental cost-effectiveness ratio as low as US $\$ 2500$ to US $\$ 19,000 /$ life-year gained $(13,14)$. The ELCAP studies have shown that: LDCT is very sensitive in the detection of early-stage lung cancer; serial scanning avoids the need for excessive invasive procedures; there are fewer false-positive scans during annual screening after a baseline scan; and CT screening may be cost effective (but not cost saving). The ELCAP initiative is now a multicentre, international collaboration, with data on thousands of patients forthcoming.

Findings similar to the ELCAP trial have been reported in observational studies published by other groups across the world $(15-18)$. Rates of malignancy, NCNs and false-positives vary depending on the age and smoking history of patients enrolled into the screening program, technology-related factors such as slice collimation and prevalence of granulomatous disease. Algorithms for follow-up of positive scans are under development and continue to be refined and re-evaluated.

Critics of lung cancer screening have suggested that LDCT screening may uncover many biologically unimportant tumours. How important is 'overdiagnosis' in lung cancer? Flehinger et al (19) studied the outcomes of 45 patients in three major CXR screening studies who did not undergo resection of a screen-detected stage 1 lung cancer. They found only two five-year survivors, suggesting that indolent lung cancers were uncommon. In the same year, Sobue et al (20) reported their retrospective review of 42 screen-detected stage 1 lung cancer patients who did not undergo surgical resection; median survival was only 25 months, with $80 \%$ of patients dying of their cancer. In medically inoperable patients with stage 1 lung cancer treated with radical radiotherapy, $75 \%$ of patients still died from a cancer-related cause, while the remainder died of intercurrent illness (21). Yankelvitz et al (22) reviewed CXR studies from the Mayo Lung Project and Memorial-Sloan Kettering Project and found doubling times of 101 and 144 days, respectively, with only $5 \%$ of cases having a doubling time longer than 400 days. In the ELCAP incidence study, doubling times ranged from 60 to 170 days in the diagnosed cases of nonsmall cell lung cancer (12). Finally, in a prospective surgical study of lobectomy versus limited resection for T1N0 disease, the limited resection arm had a higher incidence of local recurrence and cancer-specific mortality; if indolent tumours were common, the limited resection arm would have been expected to have survival rates similar to the lobectomy group (23). Collectively, these data suggest that the natural history of most lung cancers is indeed fatal and that LDCT screening does not lead to excess harm by overdiagnosis.

Demonstrating that LDCT can accurately detect early stage cancers does not translate to better a population outcome. What about survival? Previous studies have found five-year survival rates of $60 \%$ to $70 \%$ for stage $1 \mathrm{~A}$ cancers not detected by screening CT. Approximately one-half of these patients may die from distant metastases, suggesting the presence of occult, disseminated micrometastases, even in T1N0 tumours. Thus, it may be argued that the nature of lung tumour biology is such that only modest benefits can result from screening. Does this observation hold true if very small T1 lesions are detected? Gajra et al (24) retrospectively examined 246 consecutive patients with pathological stage 1A lung cancer. Median length of follow-up was five years. They found significantly improved survival in 80 patients with T size smaller than $1.6 \mathrm{~cm}$ compared with 160 patients with T size $1.6 \mathrm{~cm}$ or greater (90\% versus $80 \%, \mathrm{P}<0.05)$. Similar findings were reported by Okada et al (25) in their retrospective review of 250 patients with screendetected tumours. These data suggest that the $3 \mathrm{~cm}$ cut-off in the present staging system should be subdivided, because prognosis for small versus larger T1 tumours is different.

Ideally, results of a randomized, controlled study would be available to show a reduction in mortality in a CT-screened population compared with an unscreened group. However, a randomized, controlled trial of a screening test requires thousands of patients, many years of follow-up and millions of dollars. In the end, such a study may be open to some of the same methodological criticisms as the CXR trials. In the meantime, are we at risk of rogue CT screening programs being set up by entrepreneurial radiologists? Will patients be willing to wait for results of further trials, and will they be willing to be randomly assigned to a nonscreening arm? On the other hand, observational studies are important for giving information regarding disease prevalence, frequency of follow-up tests, complications and costs. They also allow for rapid assessment of emerging technologies, and they can be carried out over a shorter time interval at less expense. Observational studies may be valuable in supporting LDCT for screening if stage shift and survival benefits are demonstrated. Although further study is required before implementation of a mass screening program, neither patients nor their physicians may be willing to wait longer than one decade for the results of a randomized, controlled trial. The evidence presented supports the role of annual, low dose CT for lung cancer screening.

ACKNOWLEGEMENTS: Financial support was provided by a travel grant from the Canadian Thoracic Society.

\section{REFERENCES}

1. National Cancer Institute of Canada. Canadian Cancer Statistics 2003. Toronto: National Cancer Institute of Canada, 2003.

2. World Health Organization. GLOBOCAN 2000: Cancer Incidence, Mortality and Prevalence Worldwide, Version 1.0.

<http://www-dep.iarc.fr/globocan/globocan.html> (Version current at March 19, 2004)

3. Breathnach OS, Freidlin B, Conley B, et al. Twenty-two years of phase III trials for patients with advanced non-small-cell lung cancer: Sobering results. J Clin Oncol 2001;19:1734-42. 
4. Robinson LA, Wagner H Jr, Ruckdeschel JC, American College of Chest Physicians. Treatment of stage IIIA non-small cell lung cancer. Chest 2003;123(Suppl 1):202S-20S.

5. Ellis JRC, Gleeson FV. Lung cancer screening. Br J Radiol 2001;74:478-85.

6. Gotway MB, Webb WR. CT for lung cancer screening. Appl Radiol 2002;31:21-33.

7. Manser RL, Irving LB, Byrnes G, Abramson MJ, Stone CA, Campbell DC. Screening for lung cancer: A systematic review and meta-analysis of controlled trials. Thorax 2003;58:784-9.

8. Strauss GM, Gleason RE, Sugarbaker DJ. Chest x-ray screening improves outcome in lung cancer: A reappraisal of randomized trials on lung cancer screening. Chest 1995;107(Suppl 6):270S-9S.

9. Bach PB, Kelley MJ, Tate RC, McCrory MC. Screening for lung cancer: A review of the current literature. Chest 2003;123(Suppl 1):72S-82S.

10. Brown MS, Goldin JG, Suh RD, McNitt-Gray MF, Sayre JW, Aberle DR. Lung micronodules: Automated method for detection at thin-slice CT - initial experience. Radiology 2003;226:256-62.

11. Henschke CI, McCauley DI, Yankelevitz DF, et al. Early Lung Cancer Action Project: Overall design and findings from baseline screening. Lancet 1999;34:99-105.

12. Henschke CI, Naidich DP, Yankelevitz DF, et al. Early lung cancer action project: Initial findings on repeat screenings. Cancer 2001;92:153-9.

13. Wisnivesky JP, Mushlin AI, Sicherman N, Henschke CI. The cost-effectiveness of low-dose CT acreening for lung cancer: Preliminary results of baseline screening. Chest 2003;124:614-21.

14. Marshall D, Simpson KN, Earle CC, Chu CW. Economic decision analysis model of screening for lung cancer. Eur J Cancer 2001;37:1759-67.

15. Sone S, Li F, Yang Z, et al. Results of three-year mass screening programme for lung cancer using mobile low-dose spiral computed tomography scanner. Br J Cancer 2001;84:25-32.
16. Swenson SJ, Jett JR, Sloan JA, et al. Screening for lung cancer with low-dose spiral computed tomography. Am J Respir Crit Care Med 2002;165:508-13.

17. Nawa T, Nakagawa T, Kusano S, Kawasak Yi, Sugawara Y, Nakata H. Lung cancer screening using low-dose spiral CT: Results of baseline and 1-year follow-up studies. Chest 2002;122:15-20.

18. Pastorino U, Bellomi M, Landoni C, et al. Early lung-cancer detection with spiral CT and positron emission tomography in heavy smokers: 2-year results. Lancet 2003;362:593-7.

19. Flehinger BJ, Kimmel M, Melamed MR. The effect of surgical treatment on survival from early lung cancer: Implications for screening. Chest 1992;101:1013-8.

20. Sobue T, Suzuki T, Matsuda M, Kuroishi T, Ikeda S, Naruke T. Survival for clinical stage I lung cancer not surgically treated: Comparison between screen-detected and symptom-detected cases. The Japanese Lung Cancer Screening Research Group. Cancer 1992;69:685-92.

21. Sibley GS. Radiotherapy for patients with medically inoperable Stage I nonsmall cell lung carcinoma: Smaller volumes and higher doses - A review. Cancer 1998;82:433-8,

22. Yankelevitz VF, Kostis WJ, Henschke CI, et al. Overdiagnosis in chest radiographic screening for lung carcinoma: Frequency. Cancer 2003;97:1271-5.

23. Ginsberg RJ, Rubinstein LV. Randomized trial of lobectomy versus limited resection for T1 N0 non-small cell lung cancer. Lung Cancer Study Group. Ann Thorac Surg. 1995;60:615-22.

24. Gajra A, Newman N, Gamble GP, Abraham NZ, Kohman LJ, Graziano SL. Impact of tumor size on survival in stage IA non-small cell lung cancer: A case for subdividing stage IA disease. Lung Cancer 2003;42:51-7.

25. Okada M, Sakamoto T, Nishio W, Uchino K, Tsubota N. Characteristics and prognosis of patients after resection of nonsmall cell lung carcinoma measuring $2 \mathrm{~cm}$ or less in greatest dimension. Cancer 2003;98:535-41. 


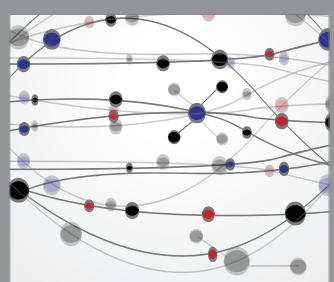

The Scientific World Journal
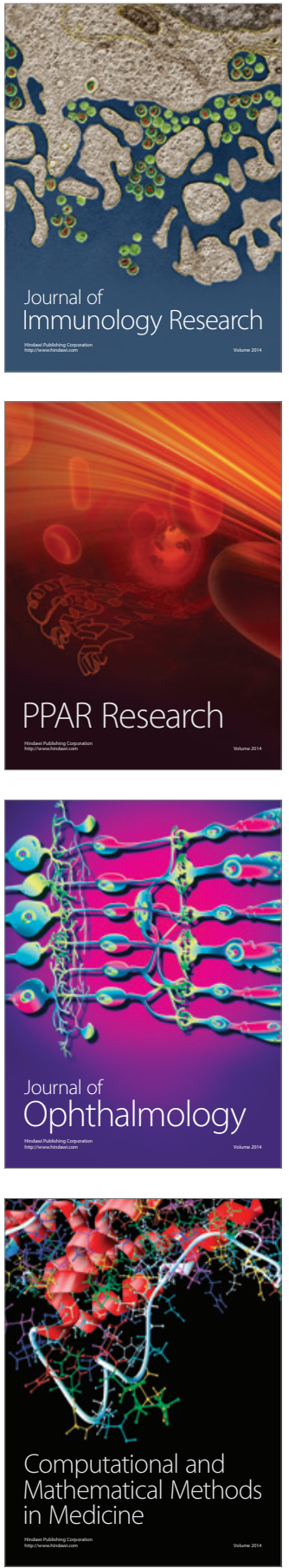

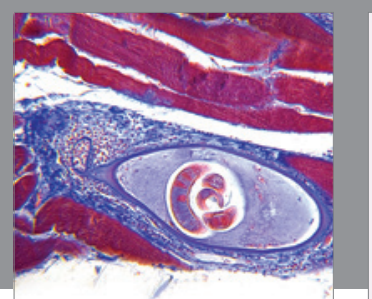

Gastroenterology Research and Practice

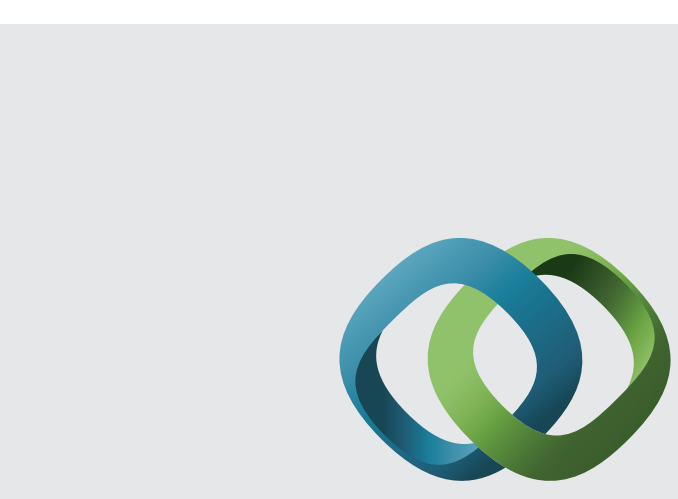

\section{Hindawi}

Submit your manuscripts at

http://www.hindawi.com
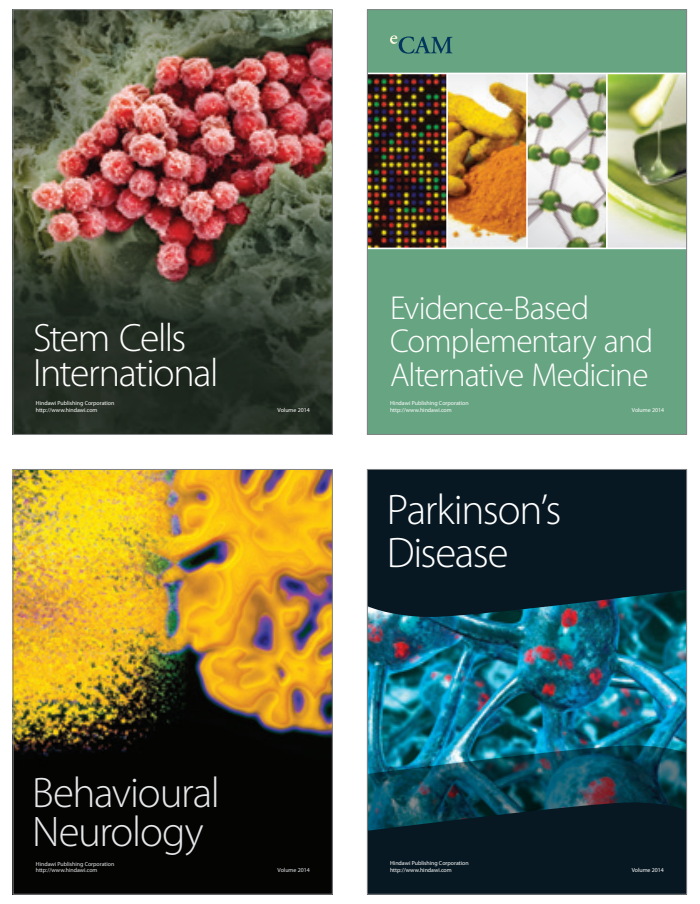
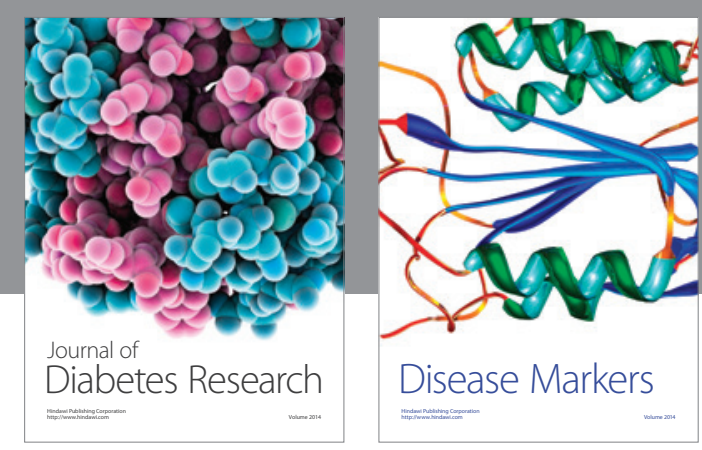

Disease Markers
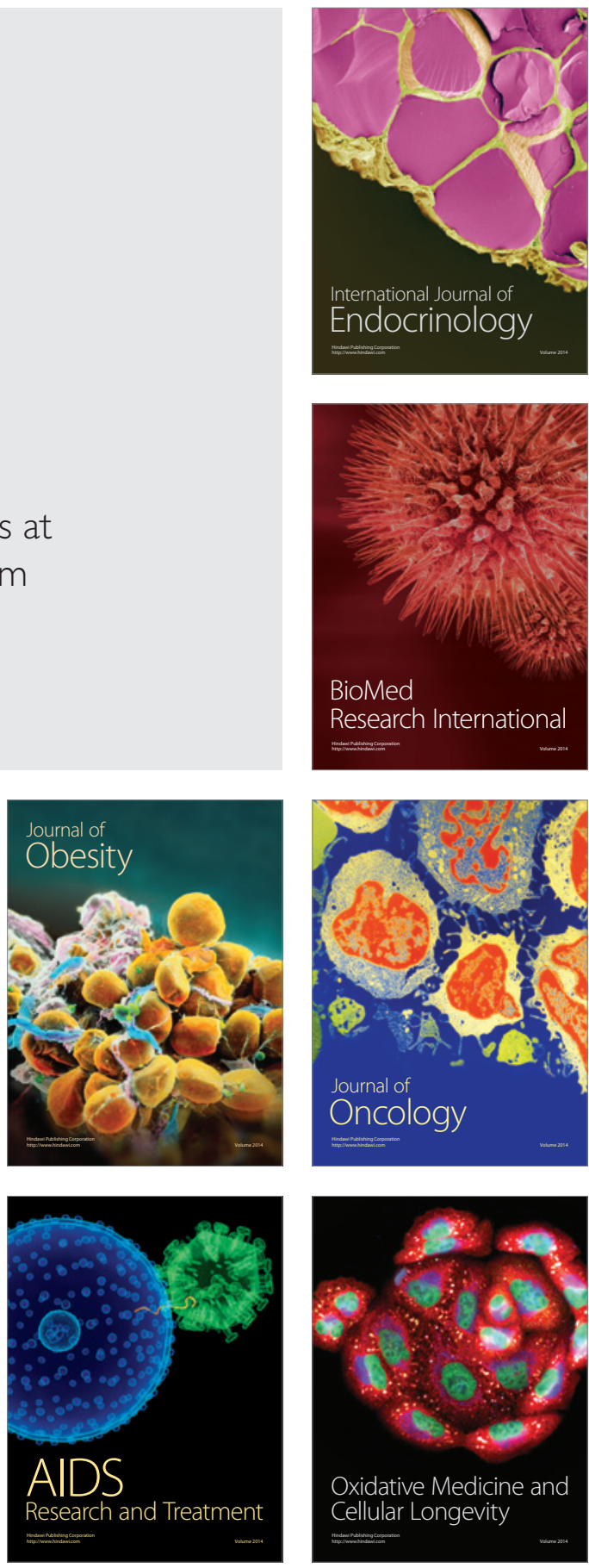